\title{
Thermal and Flow Characteristics of Discrete Double Inclined Ribs at Low Curvature Coil for GSHP Application
}

\author{
Teguh Hady Ariwibowo ${ }^{\mathrm{a}, *}$, Keishi Kariya ${ }^{\mathrm{b}}$, and Akio Miyara ${ }^{\mathrm{c}}$ \\ ${ }^{a}$ Graduate School of Science and Engineering, Saga University, Saga, Japan, teguhhady@gmail.com \\ bepartment of Mechanical Engineering, Saga University, Saga, Japan, kariya@me.saga-u.ac.jp \\ cDepartment of Mechanical Engineering, Saga University, Saga, Japan, miyara@me.saga-u.ac.jp
}

\begin{abstract}
In this study, the thermal and hydraulic characteristics in low curvature coil with Discrete Double Inclined Ribs (DDIR) were investigated. Water is selected as a working fluid, and flowrate range from 1 to $5 \mathrm{~L} / \mathrm{min}$, which is a low flowrate condition. Effect of geometry parameters such as distance between ribs, curvature ribs, and ribs inclination angles was observed. Results obtained by numerical simulations show that the heat transfer in DDIR-coil is 7.7 to $29.11 \%$ greater than that in plain coil, while the pressure drop was approximately 12.7 to $89.5 \%$ larger than that of plain-coil. A COP improvement factor that is calculated based on energy loss by pressure drop and energy saving by heat transfer enhancement was found to vary between 0.25 and 5.29. Flow visualization shows that there are two vortexes in cross-section at the downstream, and local vortexes arise around the ribs of DDIR-coil, which shows similar pattern and strength to DDIR-straight tube. The vortex makes a long fluid particle path and strengthens the turbulent mixing between the wall and the core flow of the coil. Based on these findings, DDIR-coil is recommended for applications in Slinky Ground Heat Exchangers, especially at low flowrates.
\end{abstract}

Keywords: CFD, discrete double inclined ribs, low curvature coil, longitudinal vortex

\section{Introduction}

The Ground Source Heat Pump (GSHP) has been proven as an efficient technology in the utilization of geothermal since the end of 1940s. The application of GSHP does not require high geothermal energy and can be installed in many places. This utilization is because soil temperatures have small fluctuations, while ambient air temperatures vary drastically. For example, in winter, air temperatures fluctuate from $0 \mathrm{C}$ to $16 \mathrm{C}$ [1].

Ground Heat Exchanger (GHE) is the primary key in the performance of the GHSP System. Increasing the efficiency of the GSHP system can be done by increasing the efficiency of GHE. Therefore, increasing the efficiency of the GSHP system can be done by increasing the efficiency of GHE by adopting a more advanced geometry. In general, Ground Heat Exchangers (GHE) in the GSHP systems is classified into two, namely the vertical GHE (VGHE) loop and horizontal GHE (HGHE) loop.

Popular HGHE horizontal loop configurations are straight pipe heat exchangers and slinky heat exchangers [1]. This heat exchanger requires a more extensive installation area than that of vertical GHE (VGHE). Based

${ }^{*}$ Corresponding author. Tel.: +81-707-648-6605 Graduate School of Science and Engineering, Saga University Saga, Japan, 840-8502 on studies of GHE geometry, slinky GHE is commonly utilized in HGHE application. This GHE has several advantages over vertical heat exchangers, i.e., easy installation, no need for special installation skills, and low installation costs. HGHEs are commonly set up in shallow trenches at a depth of 1 to $2 \mathrm{~m}$ from the ground surface.

To improve GHE performance, many researchers changed the tube design, some of which were on VGHE. Fin [2], and groved tube [3] were applied to improve thermal performance of VGHE. However, the modification of the geometry configuration is not commonly found in HGHE, especially the slinky type.

Several studies have been carried out to improve the performance of the slinky HGHE. Wu et al. [4] investigated some curvature coils and central coil intervals. They concluded that the difference in curvature did not show a significant increase in heat transfer extraction. However, a small curvature has a higher heat extraction rate at the same trench length. Central distance intervals increase specific heat extraction. However, increasing the central interval distance decreases the heat extraction at the same trench length. Selamat et al. [5] conducted simulation on the influence of the reclined and standing orientation of the slinky-ground heat exchanger. The results of this study indicate the use of standing orientation can extend the adequate period time by $14 \%$. Ali et al. [6] investigated the effect of standing and 
reclined orientation on the performance of slinky ground heat exchangers. They stated that the increase in heat transfer standing was higher than that of reclined. Standing slinky ground heat exchangers are influenced by deeper soil temperatures besides the amount of black fill material standing is more excellent than reclined slinky ground heat exchangers, which have higher thermal conductivity than the soil at the location.

Slinky GHE type has a low curvature coil. Hardik et al. [7] experimented with the effect of curvature on the coefficient of heat transfer on several types of coil. They claim that large curvature results in high heat transfer dute to strong secondary flow for thermal mixing. The curvature coil performance character has similarities with a straight tube. In low curvature coil, the weak centrifugal force leads to a low-pressure drop and low-heat transfer, especially at low flow rates [8]. Hence, this weakness could be solved by augmentation of coil.

Meng [9] experimented with the first Discrete Double Inclined Ribs (DDIR) straight tube study to improve heat transfer performance in the heat exchanger as his Ph.D. thesis in 2003. The result shows that the ribs could generate longitudinal vortex. $\mathrm{Li}$ et al. [10] did an experiment and simulation of the thermal characteristics of the DDIR-tube. They concluded that DDIR-straight tube could increase heat transfer increase from $100 \%$ to $120 \%$ higher than that of plain tube. However, the pressure drop has also increased from $170 \%$ to $250 \%$. Zheng et al. [11] analyzed the thermal and fluid flow performance of DDIR-tubes using numerical analysis. Based on this study, DDIR-tube can increase heat transfer and friction factor 1.58-2.46 and 1.82-5.03 times above than that of smooth tube, respectively.

We conducted preliminary studies on the effect of DDIR on the $2.22 \mathrm{~m}^{-1}$ curvature coil [12]-[15]. The result shows that in heat transfer and pressure drop increase in the increase both ribs height and flowrate. High ribs can lead to a more significant distorted vortex of flow structure than that of plain coil. The research shows that DDIR-coil generates two vortexes. This result is the opposite of the use of DDIR-straight tube, which The addition of ribs can increase the amount of vortex. This phenomenon could be caused combine among three flow, i.e., primary flow, secondary flow, and rib-induced flow. However, we do not know which flow and which geometry configurations could enhance heat exchanger performance of DDIR-coil. Based on our best knowledge, there has been no research about the application of DDIR on low curvature coil heat exchanger. The goal of this research is to present a good DDIR-coil design and to obtain the characteristics of heat transfer and fluid flow in several coil configurations for GSHP application.

\section{Materials and Methods}

\subsection{Models descriptions}

The illustration diagram of DDIR-coil used in this study is shown in Fig. 1. The coil consists of three parts, namely inlet extension, test section, and outlet extension. In all simulations, the length of the extension both inlet and outlet is $200 \mathrm{~mm}$. This extension is to ensure that flows almost fully developed and eliminates disturbance in the downstream section.

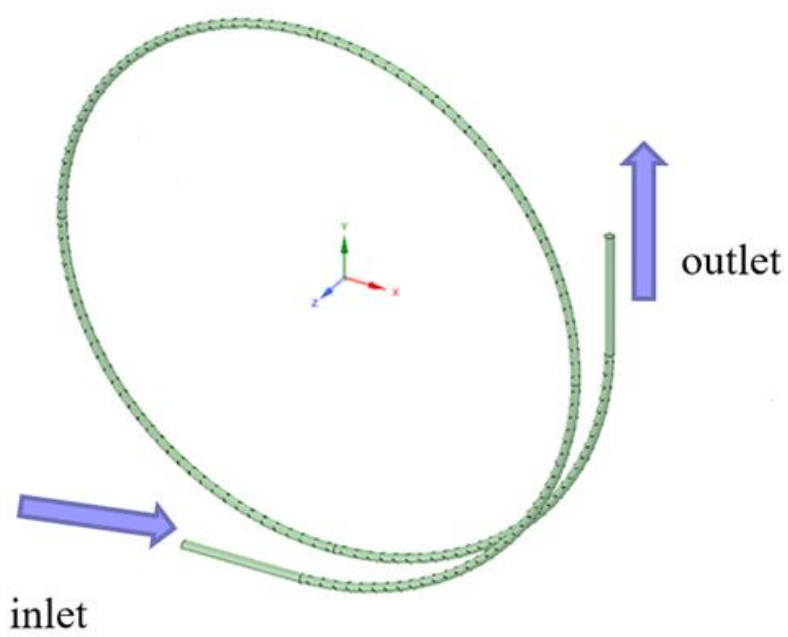

Figure 1. Schematic diagram of the DDIR-coil

The tube inside diameter, tube thickness, coil pitch, number of ribs at one perimeter are $14.46 \mathrm{~mm}, 0.71 \mathrm{~mm}$, $100 \mathrm{~mm}$, and four pieces, respectively. The geometry configuration of all models can be seen in table 1 . The curvature of coil is defined as $\mathrm{C}=1 / R$ where $R$ is the radius of coil. To find out the effect of DDIR-coil on increasing the heat exchanger performance, we also calculate straight-tube, plain-coil, and DDIR-straight tube as a benchmark. Whereas diameter of the coil, angle of ribs, curvature of coil are symbolized as $D, \alpha$, and $C$, respectively. Pitch coil, pitch ribs and ribs height are symbolized as $\mathrm{P}, \mathrm{p}$ and $\mathrm{T}$, respectively.

Table 1. Geometric specifications of the heat exchanger model

\begin{tabular}{cccccc}
\hline Model & $\begin{array}{c}\text { Curvature of } \\
\text { coil }\left(\mathbf{m}^{-\mathbf{1}}\right)\end{array}$ & $\begin{array}{c}\text { Ribs } \\
\text { Height }(\mathbf{m m})\end{array}$ & $\begin{array}{c}\text { Ribs } \\
\left.\text { Angle } \mathbf{(}^{\mathbf{0}}\right)\end{array}$ & $\begin{array}{c}\text { Ribs Pitch } \\
(\mathbf{m m})\end{array}$ & $\begin{array}{c}\text { Axial Length } \\
(\mathbf{m m})\end{array}$ \\
\hline MR-C1 & 3.33 & 1 & 45 & 22.50 & 2359 \\
MR-C2 & 2.66 & 1 & 45 & 22.50 & 2948 \\
MR-A1 & 2.22 & 1 & 20 & 22.50 & 3534 \\
MR-A2 & 2.22 & 1 & 30 & 22.50 & 3534 \\
MR-P1 & 2.22 & 1 & 45 & 33.75 & 3534 \\
MR-P2 & 2.22 & 1 & 45 & 45.00 & 3534 \\
MR-P3/MR-C3/MR-A3 & 2.22 & 1 & 45 & 22.50 & 3534 \\
MR-S & - & 1 & 45 & 22.50 & 3534 \\
MP-C1 & 3.33 & - & - & - & 2359 \\
MP-C2 & 2.66 & - & - & - & 2948 \\
MP-C3 & 2.22 & - & - & - & 3534 \\
\hline
\end{tabular}




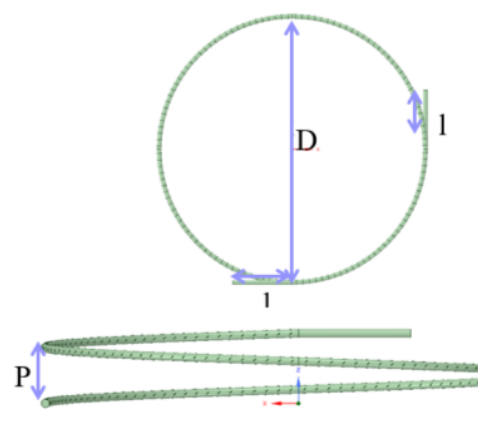

(a)

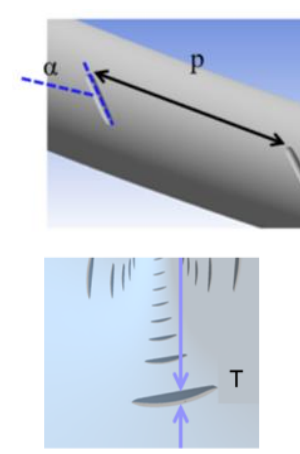

(b)
Figure 2. Structure and configuration of DDIR-coil (a) top view and side of coil, (b) location of ribs inside and outside coil surface

\subsection{Governing equations and mathematical methods}

The flow simulation utilizes Reynolds averaged Navier-Stokes (RANS) equation. The simulation is assumed as heat transfer, and fluid flow is turbulent, steady-state, and no heat loss to environment. Shear stress tensor $\kappa-\omega(\mathrm{SST} \kappa-\omega)$ is a turbulent model that shows an excellent performance in modeling adverse pressure gradient. This model combines $\kappa-\omega$ model in near-wall and $\kappa-\varepsilon$ model in the core flow [16].

The Governing equations are as per the following Continuity equation:

$$
\frac{\partial\left(\rho u_{i}\right)}{\partial x_{i}}=0
$$

Momentum equation:

$$
\begin{gathered}
\frac{\partial\left(\rho u_{i}\right)}{\partial t}+\frac{\partial\left(\rho u_{i} u_{j}\right)}{\partial x_{j}}= \\
-\frac{\partial p}{\partial x_{i}}+\frac{\partial}{\partial x_{j}}\left(\mu \frac{\partial\left(u_{i}\right)}{\partial x_{j}}-\rho \overline{u_{\imath}^{\prime} u_{J}^{\prime}}\right)
\end{gathered}
$$

Energy equation:

$$
\frac{\partial}{\partial x_{i}}(\rho T)+\frac{\partial}{\partial x_{i}}\left(\rho u_{i} T\right)=\frac{\partial}{\partial x_{i}}\left(\frac{\lambda}{c_{p}} \frac{\partial T}{\partial x_{i}}\right)
$$

Turbulence kinetic energy equation:

$$
\begin{gathered}
\frac{\partial(\rho \kappa)}{\partial t}+\frac{\partial\left(\rho u_{i} \kappa\right)}{\partial x_{i}}= \\
\tilde{P}_{\kappa}-\beta^{*} \rho \kappa \omega+\frac{\partial}{\partial x_{i}}\left(\left(\mu+\sigma_{\kappa} u_{t}\right) \frac{\partial \kappa}{\partial x_{i}}\right)
\end{gathered}
$$

Specific dissipation rate equation:

$$
\begin{gathered}
\frac{\partial(\rho \omega)}{\partial t}+\frac{\partial\left(\rho u_{i} \omega\right)}{\partial x_{i}}=\varphi \rho S^{2}-\beta \rho \omega^{2}+ \\
\frac{\partial}{\partial x_{i}}\left(\left(\mu+\sigma_{\omega} u_{t}\right) \frac{\partial \omega}{\partial x_{i}}\right)+ \\
2\left(1-F_{1}\right) \rho \sigma_{\omega^{2}} \frac{1}{\omega} \frac{\partial \kappa}{\partial x_{i}} \frac{\partial \omega}{\partial x_{i}}
\end{gathered}
$$

where blending function $F_{1}$ is described as follows

$$
F_{1}=\tanh \left\{\left\{\min \left[\max \left(\frac{\sqrt{\kappa}}{\beta^{*} \omega y}, \frac{500 v}{y^{2} \omega}\right), \frac{4 \rho \sigma_{\omega 2} \kappa}{C D_{\kappa \omega} y^{2}}\right]\right\}^{4}\right\}
$$

where

$$
C D_{\kappa \omega}=\max \left(2 \rho \sigma_{\omega 2}(1 / \omega)\left(\partial \kappa / \partial x_{i}\right)\left(\partial \omega / \partial x_{i}\right), 10^{-10}\right)
$$

and $\mathrm{y}$ is the distance to the adjacent wall. Turbulent eddy viscosity is described by

$$
v_{t}=\frac{\varphi_{1} \kappa}{\max \left(\varphi_{1} \omega, S F_{2}\right)}
$$

where $\mathrm{S}$ is a constant measure of strain rate and $F_{2}$ is a second blending function described as follows

$$
F_{2}=\left\{\left[\max \left(\frac{2 \sqrt{\kappa}}{\beta^{*} \omega y}, \frac{500 v}{y^{2} \omega}\right)\right]^{2}\right\}
$$

The SST model uses a production limiter to prevent turbulence buildup in stagnation areas as follows

$$
P_{\kappa}=\mu_{t} \frac{\partial u_{i}}{\partial x_{j}}\left(\frac{\partial u_{i}}{\partial x_{j}}+\frac{\partial u_{j}}{\partial x_{i}}\right) \rightarrow \tilde{P}_{\kappa}=\min \left(P_{\kappa}, 10 \cdot \beta^{*} \rho \kappa \omega\right)
$$

All constants used, $\beta^{*}, \varphi_{1}, \beta_{1}, \sigma_{\kappa l}, \sigma_{\omega 1}, \varphi_{2}, \beta_{2}, \sigma_{\kappa 2}$, and $\sigma_{\omega 2}$, are a combination of constants calculated based on the $\kappa-\varepsilon$ and $\kappa-\omega$ model. All equations were solved using ANSYS FLUENT 17.2, which is a commercial software based on the finite volume method. For Velocity-Pressure Coupling, the SIMPLE algorithm was utilized. The minimum convergence criterion for the continuity, velocity, and turbulence equation is $10^{-3}$, and the energy equation is $10^{-7}$.

\subsection{Grid generation and independence test}

Three-dimensional mesh made using ANSYS Meshing 17.2. The discretized domain uses unstructuredtetrahedral elements, as shown in Fig. 3. To obtain more accurate results, the value of $\mathrm{y}^{+}$is less than 1 . This setting can make fine quality mesh near the wall and ribs. To find out the accuracy of numerical simulations, we use three sets of grids with 16136244 (coarse), 17779526 (medium), and 20175527 (fine) elements, which were used to test the grid independence test at $\operatorname{Re}=4115$. In this test, the pressure drop and heat transfer rate were checked. The relative deviations of pressure drop and heat transfer rate were determined based on the value of the fine mesh. Nusselt numbers of coarse, medium, and fine mesh were $71.27,72.02$, and 72.76, respectively. The deviation of Nusslet number from coarse to fine and medium to fine were 2.04 and $1.01 \%$, respectively. Friction factors of coarse, medium, and fine mesh were 0.08273, 0.08330, and 0.08391 , respectively. The deviation friction factors from coarse to fine and medium to fine are 1.39 and 0.71 $\%$, respectively. Therefore, the 20175527-mesh system was chosen quite dense in this study. 


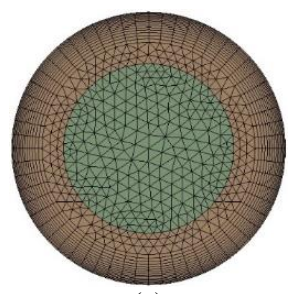

(a)

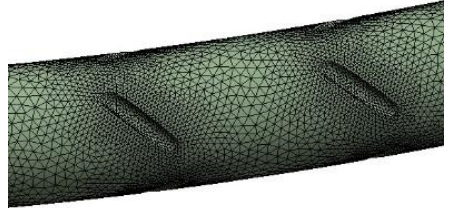

(b)
Figure 3. Mesh system of DDIR-coil: (a) mesh in the cross-section plane of coil, (b) mesh on ribs

\subsection{Boundary condition and data reduction}

Velocity inlet boundary condition was adopted with uniform velocity, water temperature constant value is 280 $\mathrm{K}$, the pressure outlet boundary condition was utilized. The flow at the wall was assumed as no slip. The wall temperature is assumed to be constant and uniform at 291 $\mathrm{K}$ in the inner side of the tube. Outside of the tube was assumed as perfectly insulated, hence no heat loss to surroundings. The tested flow rate is $1 \mathrm{~L} / \mathrm{min}(R e=1028)$, $2 \mathrm{~L} / \min (R e=2057), 3 \mathrm{~L} / \min (R e=3086), 4 \mathrm{~L} / \min (R e=$ $4115)$, and $5 \mathrm{~L} / \mathrm{min}(R e=5144)$. The equation of $\kappa$ in the $\kappa-\omega$ turbulence model uses enhanced wall treatment in wall boundary conditions. This setting means that for all boundary conditions for fine mesh, Low-Reynoldsnumber treatment was utilized. To find out the strength of vortex, we use swirl strength $\left(\lambda_{c i}\right)$. This method calculates strength of vortex based on the velocity gradient tensor. Swirl strength uses the imaginary portion of the complex eigenvalues of the velocity gradient tensor to describe vortices [17], [18].

The following equation calculates heat transfer rate of the coil

$$
Q=\dot{m} c_{p}\left(T_{o}-T_{i}\right)
$$

where, $T_{o}$ and $T_{i}$ are bulk temperature on downstream and upstream crossection, respectively. Calculation of the average heat transfer coefficient is obtained as follows

$$
h=\frac{Q}{\int_{A} d A \Delta T_{m}}
$$

where, $\Delta T_{m}$ is Logarithmic Mean Temperature Difference (LMTD) and $T_{w}$ is wall temperature.

$$
\Delta T_{m}=\frac{\left(T_{w}-T_{o}\right)-\left(T_{w}-T_{i}\right)}{\ln \left(\frac{T_{w}-T_{o}}{T_{w}-T_{i}}\right)}
$$

All water properties are assumed to be constant, so the use of LMTD calculations is valid. Some dimensionless numbers are used in this study as follows

$$
\begin{aligned}
& R e=\frac{\rho u d}{\mu} \\
& N u=\frac{h d}{\lambda}
\end{aligned}
$$

$$
f=\frac{\Delta p}{\left(\frac{l}{d}\right)\left(\rho \frac{v^{2}}{2}\right)}
$$

We use the critical Reynolds number calculation in coil, which is proposed by Ito [19]. The critical Reynolds number is shown as follows

$$
R e_{c r}=20000\left(\frac{d}{D}\right)^{0.32}
$$

Coefficient of Performance (COP) improvement factor is proposed by Jalaluddin and Miyara [20]. This parameter is to see the improvement of GSHP system due to GHE modification. The COP improvement factor is described as follows

$$
\frac{Q_{H}^{\prime}}{Q_{H}}-\frac{V \Delta p}{Q_{H}} \frac{\Delta p^{\prime}}{\Delta p}>0
$$

Where, $Q_{H}, Q_{H}^{\prime}, V, \Delta p^{\prime}$, and $\Delta p$ are heat transfer rate $(\mathrm{W} / \mathrm{m})$, an increase of heat transfer rate $(\mathrm{W} / \mathrm{m})$, volumetric flowrate $\left(\mathrm{m}^{3} / \mathrm{s}\right)$, an increase of pressure drop $(\mathrm{Pa} / \mathrm{m})$, and pressure drop $(\mathrm{Pa} / \mathrm{m})$, respectively. If left term is larger than 0 , it means the performance of GSHP system ground heat increase. The base of COP improvement factor is heat transfer and pressure drop of smooth straight tube on several corelations.

A few Nusselt number and friction factor correlations were used in the turbulent, transition, and laminar regime to determine the heat transfer rate and pressure drop for smooth straight tubes because the Reynolds number in this study was from 1028 to 5144 . Critical Reynolds number for ribs pitch variation and ribs angle is 5332. Then, critical Reynold numbers for curvature 2.22, 2.66 and 3.33 $\mathrm{m}^{-1}$ are 5332, 5652, and 6071, respectively.

For laminar region, Nusselt number and friction factor were calculated as follows

$$
\begin{aligned}
\mathrm{Nu} & =3.66 \\
f & =\frac{64}{R e}
\end{aligned}
$$

Meanwhile, the calculation of the Nusselt number in the transition region adopted a linear interpolation method of the New Gnileniski Nusslet number [21], which is valid at $2300<R e<4000$ and constant wall temperature.

$$
N u=(1-\gamma) N u_{l a m, 2300}+\gamma N u_{t u r b, 4000}
$$

where

$$
\gamma=\frac{R e-2300}{2300-4000}
$$

$N u_{\text {lam,2300 }}$ was calculated as follows

$$
\begin{gathered}
N u_{m, T}=\left(N u_{m, T, 1}^{3}+0.7^{3}+\left(N u_{m, T, 2}-0.7\right)^{3}\right. \\
\left.+N u_{m, T, 3}^{3}\right)^{1 / 3}
\end{gathered}
$$


where,

$$
\begin{aligned}
N u_{m, T, 1} & =3.66, \\
N u_{m, T, 2} & =1.615 \sqrt[3]{\operatorname{Re} \operatorname{Pr} d / L}, \\
N u_{m, T, 3} & =\left(\frac{2}{1+22 \operatorname{Pr}}\right)^{1 / 6}(\operatorname{Re} \operatorname{Pr} d / L)
\end{aligned}
$$

then $N u_{t u r b, 4000}$ was calculated as follows

$$
\begin{gathered}
N u=\frac{(f / 8)(R e-1000) \operatorname{Pr}}{1+12.7 \sqrt{f / 8}\left(\operatorname{Pr}^{2 / 3}-1\right)} \\
{\left[1+\left(\frac{d}{L}\right)^{2 / 3}\right] K}
\end{gathered}
$$

the $K$ factor was described as

$$
K=\left(\frac{P r}{P r_{w}}\right)^{0.11}
$$

The friction factors in Eq. (18) use Konakov's friction factors as follows

$$
f=\left(1.8 \log _{10} R e-1.64\right)^{-2}
$$

whereas in the transitional regime, Abraham friction factor [22] that is valid Reynold number from 2300 to 4500 are used as follows

$$
\begin{gathered}
f=30310^{-12}-3.6710^{-8} R e^{2}+ \\
1.4610^{-4} R e-0.151
\end{gathered}
$$

For the turbulent regime, calculation the Nusselt number in Eq. (18) and the friction factor use Petukhov friction factors as follows

$$
f=(0.79 \ln (R e)-1.64)^{-2}
$$

\section{Results and Discussions}

\subsection{Model validation}

We adopted some of the results of experiments conducted by Meng [9] to validate numerical simulations as shown in Fig. 4 and 5. The numerical results were following the experimental results on the f and $\mathrm{Nu} / \mathrm{Pr}^{1 / 3}$, Although there were some deviations. The average deviation between simulation and experimental results is $10.6 \%$ for $N u / \operatorname{Pr}^{1 / 3}$ and $3.9 \%$ for $f$. This deviation can be caused by the uncertainty of the experimental measurements and the difference between numerical and experimental methods. Accordingly, the numerical method used in the study of heat transfer and pressure drop is reliable.

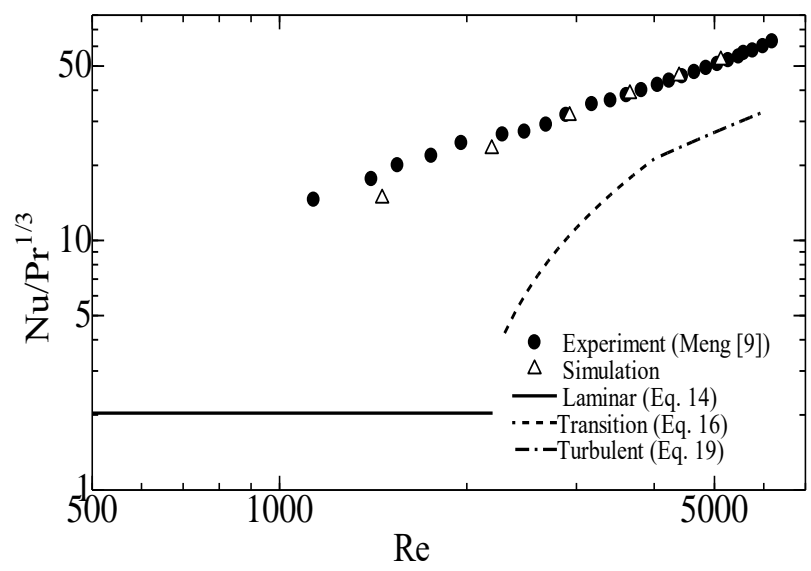

Figure 4. Comparison of $N u / \mathrm{Pr}^{1 / 3}$ between simulation results and experiment results for the proposed DDIR-straight tube

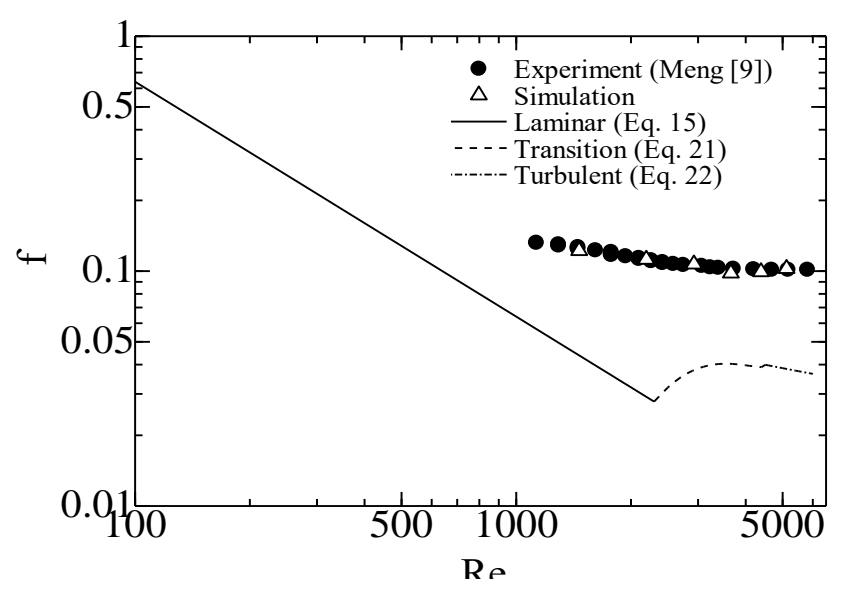

Figure 5. Comparison of friction factors between simulation results and experiment results for the proposed DDIR-straight tube

\subsection{Flow structure and heat transfer}

The behavior of the flow structure on DDIR-coil needs to be examined before discussing the results. The resulting flow analysis is an effective way of describing the flow in the DDIR-coil. Plain-coil is used as a comparison of how significant the effect of DDIR-coil is on the flow structure. Figure 6 shows a three-dimensional flow display in the downstream coil area for $\mathrm{Re}=4115$. In plain-coil, flow particles tend to occupy an outer side -coil position. The flow is caused by centrifugal force. This centrifugal force also causes some water particles near the wall to move towards the inner side of coil. In general, DDIR-coil produces two vortices when water particles pass through the ribs, namely the front vortex and the rear vortex.

In DDIR-coil, flow particles tend to be distributed almost evenly both at inner side and outer side of coil. The ribs-induced flow is caused by force of the front vortex and rear vortex, which affect the centrifugal coil force. This phenomenon is confirmed by the tendency of water particle flow near the wall from outer side of coil to be distorted to outerside of coil several times when it crosses ribs before finally, the particle flow moves towards the inner side of coil. The path of water particles in DDIR-coil tends to be longer than that of plain-coil. Tangential 
velocity and streamline observations are a remarkable way to determine the performance of DDIR effects in the flow field.

Figure 7 shows the tangential velocity vector in the downstream coil. In plain-coil, the gradient velocity vector near the wall looks smaller than in DDIR-coil. Besides, the plain-coil location of the stagnation point and separation point tends to have a longer circumferential length than that of DDIR-coil. This tendency is possible because the flow generated by ribs can distort the flow so that it shifts the location of the stagnation point and the separation point. The DDIR-straight tube shows that there are two pairs of vortices in opposite directions. The velocity gradient was significant in the two pairs of vortexes. The stagnation point and flow separation are clearly symmetrical to each other.

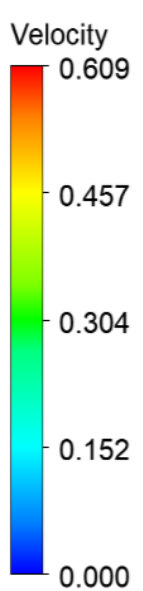

$\left[\mathrm{m} \mathrm{s}^{\wedge}-1\right]$

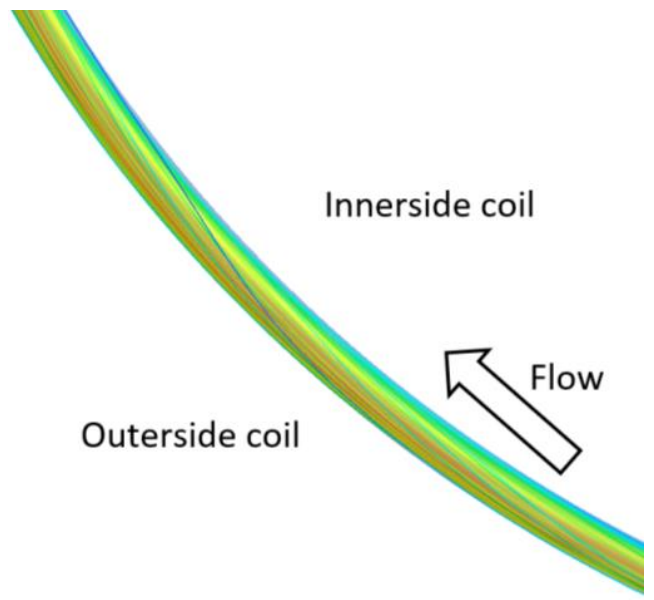

(a)

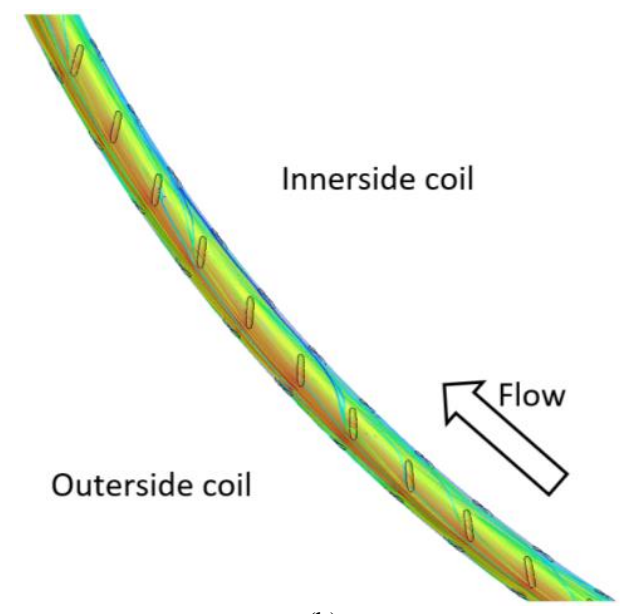

(b)

Figure 6. Limiting 3D isometric view streamline for $\mathrm{Re}=4115$ (a) MP-C3, (b) MR-C3

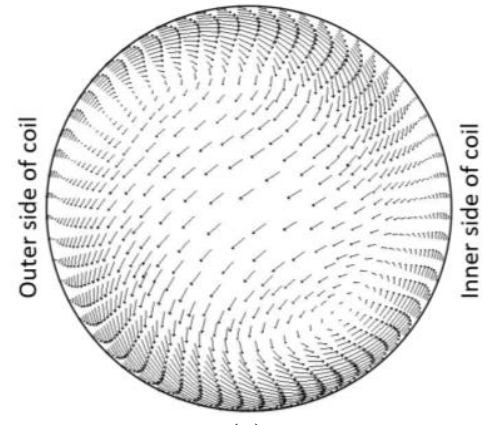

(a)

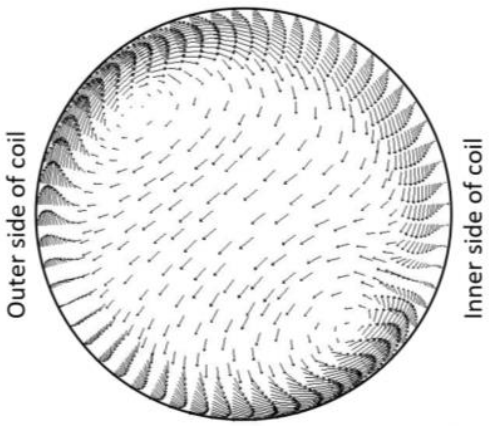

(b)

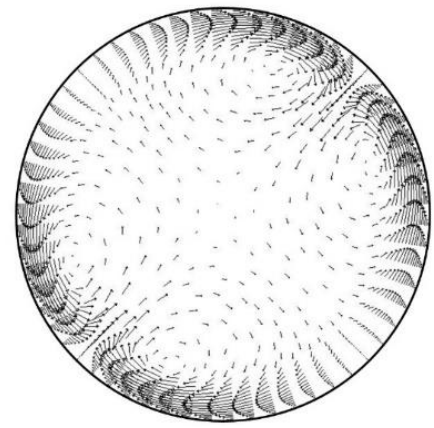

(c)

Figure 7. Tangential velocity vector in outlet test section for Re $=4115$ (a) MP-C3, (b) MR-C3, (c) MR-S

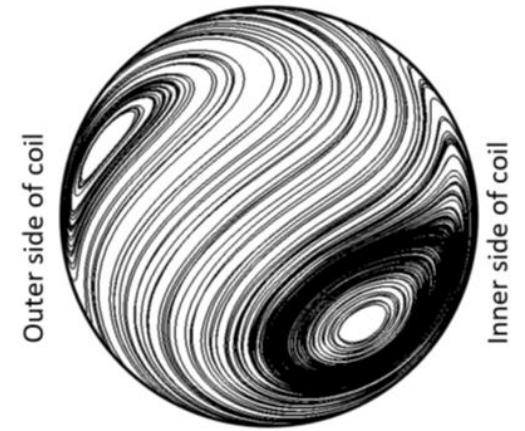

(a)

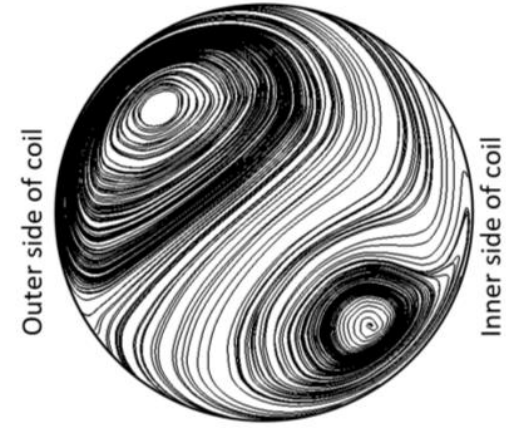

(b)

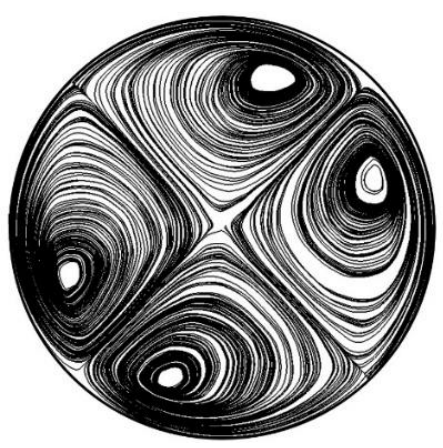

(c)

Figure 8. Streamlines in outlet test section for Re $=4115$ (a) MP-C3, (b) MR-C3, (c) MR-S 


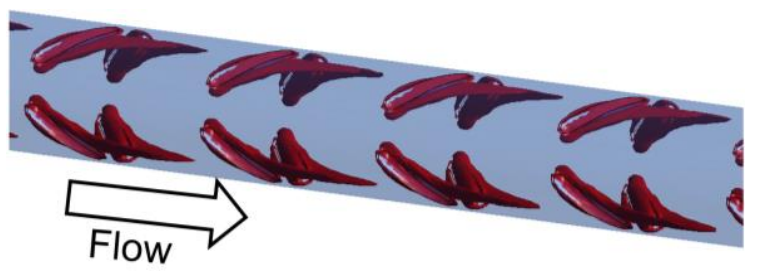

(a)

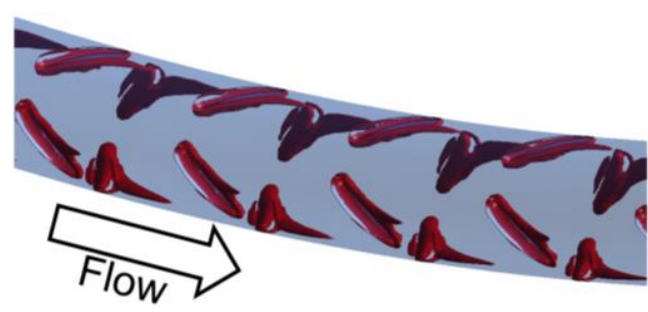

(b)

Figure 9. Vortex cores $\left(\lambda_{c i}=40 \mathrm{~s}^{-1}\right)$ generated for $\mathrm{Re}=4115$ (a) MR-S, (b) MR-C3

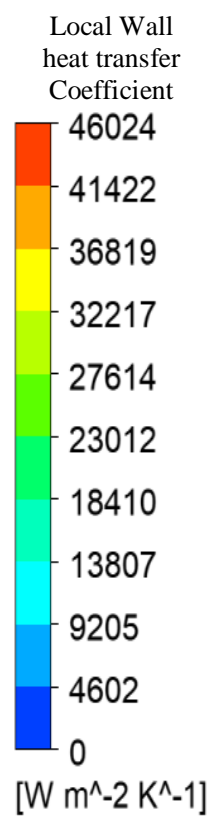

Local Wall

heat transfer

46024

27614

13807

9205

$\left[\mathrm{W} \mathrm{m}^{\wedge}-2 \mathrm{~K}^{\wedge}-1\right]$

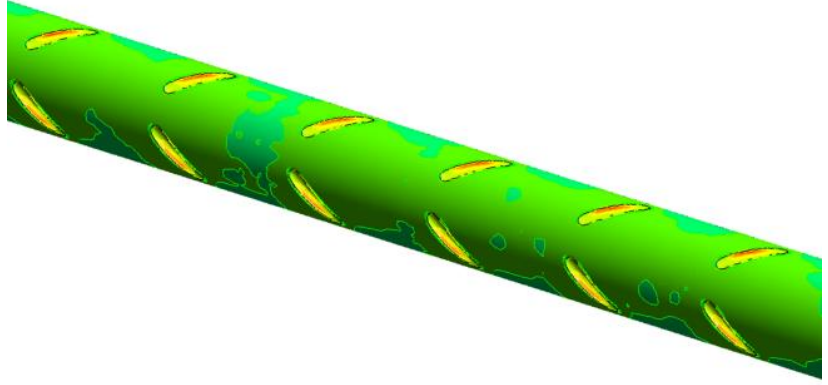

(a)

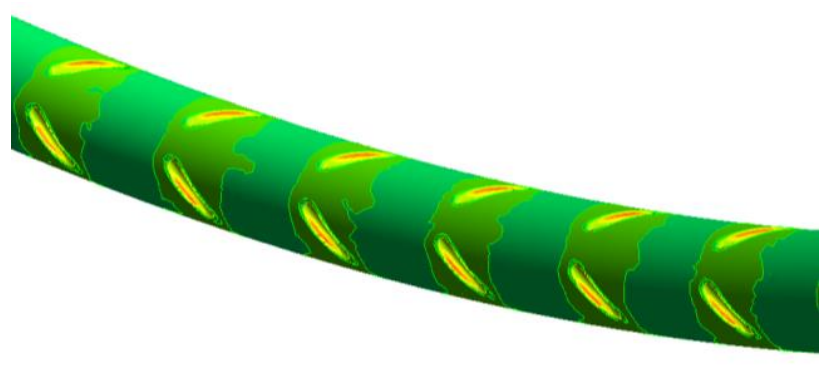

(b)

Figure 10. Local wall heat transfer coefficient contours on the wall of the test section for $\operatorname{Re}=4115$ (a) MR-S, (b) MR-C3

Figure 8 shows a streamlined downstream coil. A pair of counter-rotating vortex is produced in both plain-coil and DDIR-coil. Based on research into the application of DDIR on straight tubes, the addition of ribs is accompanied by an increase in the number of longitudinal vortices Zheng et al [11]. This research conduct by using two pairs of V-type ribs on circumferential of tube. The generated vortex should be two pairs. However, in this study, we found one pairs. No additional vortex appears because the vortex generated by the ribs joins the vortex caused by the centrifugal coil force.

This result is confirmed by the size of the vortex seen in the streamline. DDIR-coil tends to have a larger vortex size than that of plain-coil. On the DDIR-straight tube, two pairs of vortexes of similar size and flow patterns that appear dense are seen. This pattern indicates that the DDIR-stright tube pathway particles are longer than that of DDIR-coil and DDIR-plain. The quantitative increment of DDIR-coil in heat transfer and fluid flow can be seen in Fig. 11.

The movement of the ribs-induced vortex with different types of shape is illustrated in Fig. 9. Vortex core visual images are displayed based on iso-surface values of swirling strength of $40 \mathrm{~s}^{-1}$. The structure of powerful vortexes is mostly constructed behind the ribs. The structure indicate that the strength of the rear vortex is greater than that of the front vortex. On DDIR-straight tube, the distribution of the vortex appears evenly on each ribs at the same circumferential perimeter. However, on DDIR-coil, the vortex distribution is not the same in every ribs at one circumferential perimeter. This distribution is because the DDIR-straight tube primary flow and the ribs induced flow do not interfere with each other whereas the DDIR-secondary secondary flow and ribs induced flow interfere with each other.

Figure 10 shows the distribution of local heat transfer coefficient for $\mathrm{Re}=4115$ for DDIR-straight tube and DDIR-coil at the same axial length. It is clear that the heat transfer coefficient of DDIR-straight tube is slightly higher than that pf DDIR-coil on the surface of the ribs, this is because the flow is incident directly onto the surface of the ribs. The local heat transfer coefficient was discovered somewhat higher at the rear ribs due to rear induced vortex. This phenomenon is caused by the strength of the rear vortex higher than that of the front vortex. However, it can be seen clearly that the local heat transfer coefficient is slightly higher in the DDIR-straight tube than that of in the DDIR- coil. DDIR-straight tube 
produces two flows, primary flow, and ribs induced flow. Meanwhile, DDIR-coil produces three flows, namely primary flow, secondary flow, and ribs-induced flow. Secondary flow and ribs-induced flow probably combine, then weakening the strength of the vortex, hence local heat transfer in DDIR-coil is slightly smaller than that of DDIR-straight tube.

\subsection{Rib angle effect}

The variation of heat transfer rate, pressure drop and COP improvement factors with different rib angle and flowrate are shown in Figs. 11 (a), (b) and (c). As can be seen, heat transfer and pressure drop increase with increasing flow rate. This increment may be due to increased flowrate that can generate greater windward, leeward, and higher swirl strength. An interesting pattern is shown by an angle of $20^{\circ}$ and $45^{\circ}$. These two angles show almost a similar performance in both heat transfer

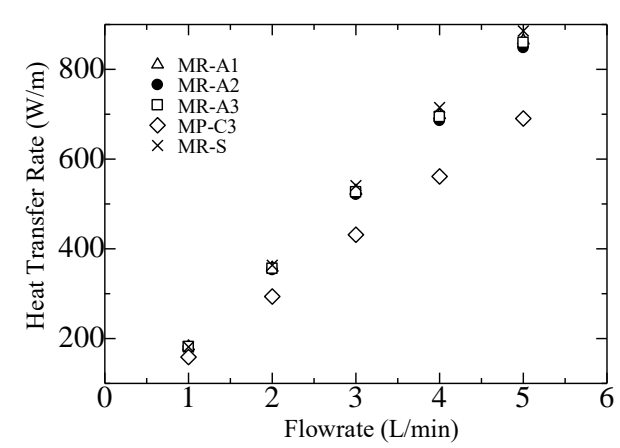

(a) and pressure drop in the range flowrate. A more detailed check shows that the $30^{\circ}$ angle has a slightly smaller heat transfer performance than that of the other angles. Whereas the pressure drop showed a more significant increase in each angle of the ribs. The increase in heat transfer is approximately $13.9 \%-25.15 \%$ and the pressure drop increases approximately $26.8 \%-89.5 \%$ over plaincoil. In Figs. 11 (a), (b) and (c), heat transfer rate, pressure drop and COP improvement factor of straight DDIRstraight tube at $45^{\circ}$ ribs angle are also plotted. The straight tube is used as benchmark performance of DDIR-coil at $45^{\circ}$. As mentioned above, DDIR is more effective in straight tube than coil. However, According to $\mathrm{Wu}$ et al. [4] coil is still more suitable for use in ground heat exchangers because the ground heat exchanger is installed in a trench and the coil has higher performance than that of straight tube in the same length of trench.

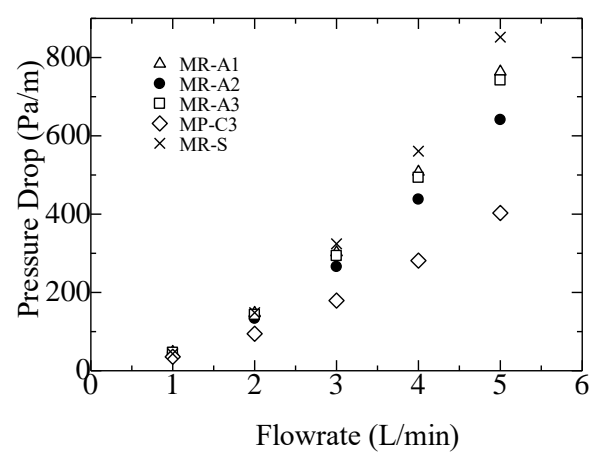

(b)

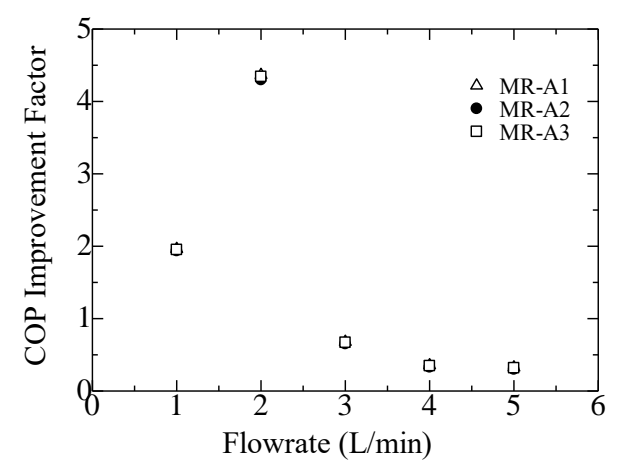

(c)

Figure 11. Performance characteristics in angle ribs variation (a) heat transfer rate, (b) pressure drop, (c) COP improvement factor

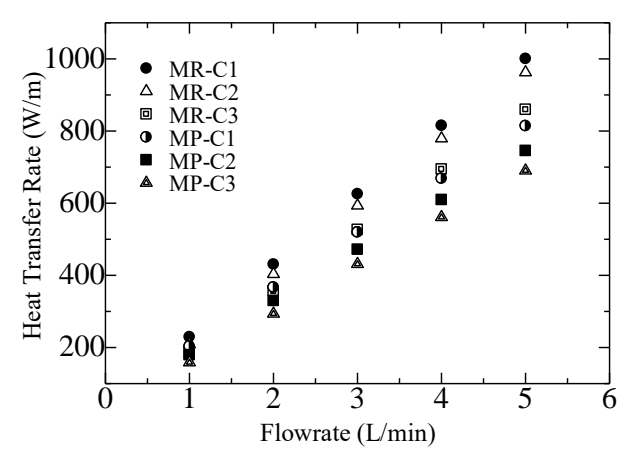

(a)

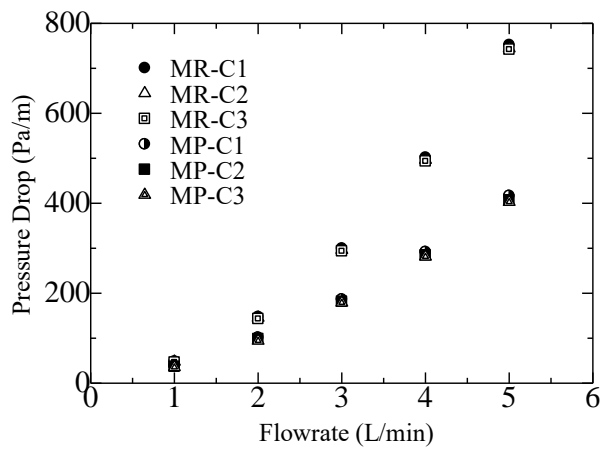

(b) 


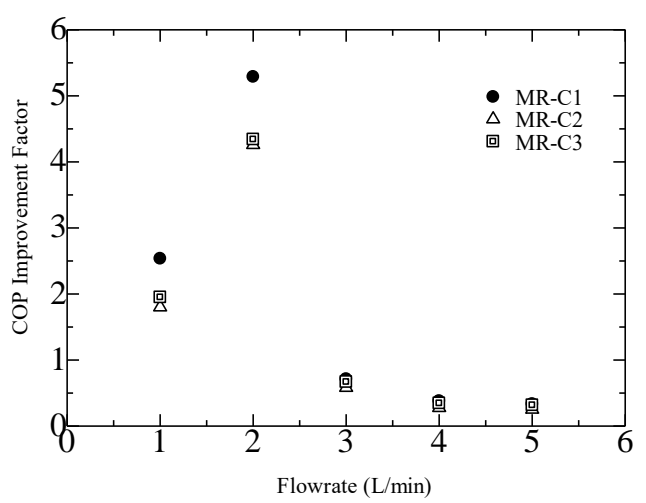

(c)

Figure 12. Performance characteristics in curvature variation (a) heat transfer rate, (b) pressure drop, (c) COP improvement factors

\subsection{Curvature effect}

Figures 12 (a), (b) and (c) show the effect of the curvature of the DDIR-coil on the performance of heat transfer, pressure drop and COP improvement factors at a rib angle of $45^{\circ}$ and pitch rib of $22.5 \mathrm{~mm}$ in flowrate from 1 to $5 \mathrm{~L} / \mathrm{min}$. The graphs also illustrate plain-coil performance as a benchmark for increasing DDIR-coil performance. Commonly, decrease in curvature on DDIRcoil results in an increase in heat transfer rate and pressure drop. The decrease in curvature appears significantly at the heat transfer rate, but the decrease in pressure drop is very insignificant. The use of DDIR-coil shows more significant than that of plain-coil. The increase in heat transfer rate on curvature $2.22,2.66$, and $3.33 \mathrm{~m}^{-1}$ are $12.70-22.85 \%, \quad 16.27-29.11 \%$, and $14.50-24.51 \%$, respectively. The increment in pressure drop on curvature

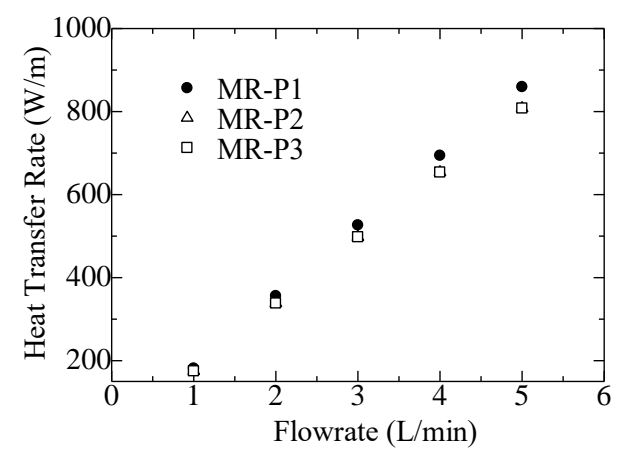

2.22, 2.66, and $3.33 \mathrm{~m}^{-1}$ are $27.28-80.45 \%, 28.87-82.81 \%$ and $30.83-84.17 \%$, respectively. The value of COP Improvement Factors varies from 0.25-5.29. This trend shows that DDIR-coil provides benefits in terms of energy conservation. Although the heat transfer rate and pressure drop characters increase with the increase in the curvature coil, the COP Improvement factor shows an interesting trend. The highest increase occurred at $2.22 \mathrm{~m}^{-1}$, and the lowest occurred at $2.66 \mathrm{~m}^{-1}$. Dramatic increase and decrease of COP Performance factors in Curvature variations also happen at first flowrate. The COP Improvement Factors tend to change drastically in flow rate 1-3 L/min because the factors are calculated on Eqs. (13)-(21) for straight tube. The trend is triggered mainly flow on the straight tube in the transition regime. Hence, drastic change happens on this area..

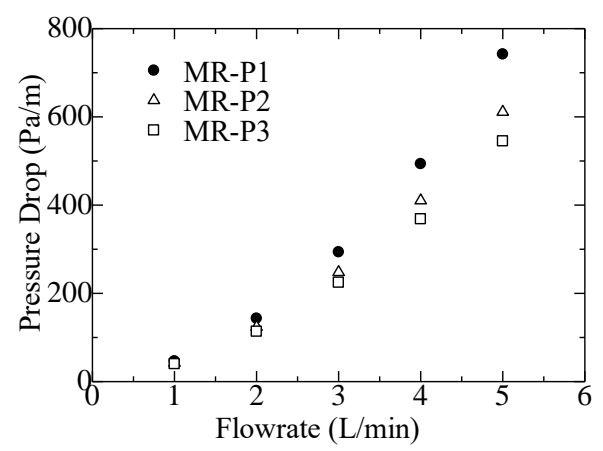

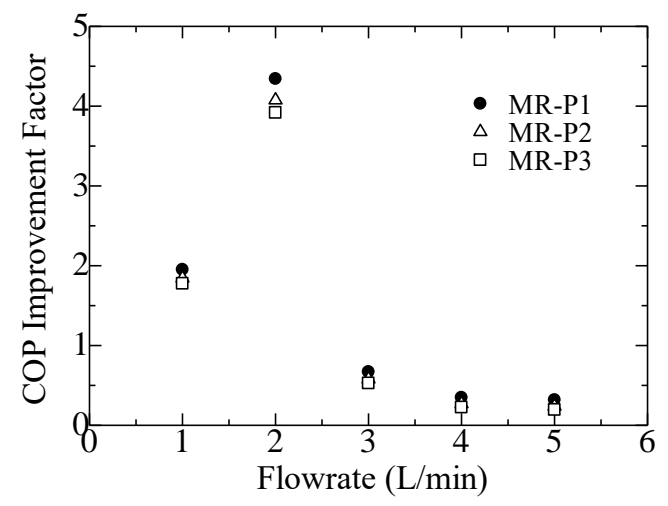

Figure 13. Performance Characteristics in ribs pitch (a) heat transfer rate, (b) pressure drop , (c) COP improvement factor 


\subsection{Ribs pitch effect}

Figures 13(a), (b) and (c) show the effect of pitch ribs on the performance of heat transfer and flow at a rib angle of $45^{\circ}$ and axial coil length of $3533.75 \mathrm{~mm}$. Pitch ribs are calculated based on the axial distance on the center coil. It is clear that the heat transfer rate and pressure drop slightly increased when pitch ribs increased. The reason for this phenomenon is that heat transfer increases more in the downstream ribs than that of upstream ribs, and increment ribs pitch decreases regions in downstream. Heat transfer and pressure drop increase, relative to plain coil, increases by $7.7-25.5 \%$ and by $12.7-84.1 \%$, respectively

The COP Improvement Factor in pitch ribs varies between 0.19 to 4.34 . It can be seen that the value of COP Improvement Factors increases with the decrease in distance between ribs on all flowrates. Significant increase in COP Improvement Factor at a flow rate of 1-2 L / min. Then there is a significant decrease in the flow rate of 2-3 $\mathrm{L} / \mathrm{min}$. Then, a decrease occurs slowly at a flow rate of 3-5 L / min. A sudden change in COP Factors that occurs at flow rates of 1-3 L / min occurs due to performance calculations occurring on a straight tube. At this flow rate, the flow is in the transition area so that the heat transfer and pressure drop have increased significantly. However, when it has passed the transition regime, tube performance tends to be more stable and turbulent enough so that straight tube performance approaches coil performance. This characteristic tends to result in a gradual decrease in COP Improvement factors at 3-5 L / min.

\section{Conclusion}

The results of the research on the effect of DDIR of Low curvature coil on increasing heat transfer, pressure drop, and COP Improvement factors are presented in this paper. The flow structure is described and analyzed. The influence of ribs angle, curvature, and ribs pitch are examined at a specific flowrate range so that the thermal and hydraulic characteristics of DDIR-coil are summarized as follows:

In the flowrate range investigated, DDIR-coil heat transfer increased from 7.7 to $29.11 \%$ bigger than that of plain-coil, and the pressure drop increased from 12.7 to $89.5 \%$ higher than that of plain-coil. COP Improvement factors approximately vary between 0.25 and 5.29.

The multiple longitudinal vortex arrangement in the DDIR-coil down stream is not apparent when compared to DDIR-straight. However, the Vortex local DDIR in coil is formed and has a strength similar to the vortex local DDIR in straight tube. The combination of secondary flow and ribs induced flow occurs so that the strength of the vortex in DDIR-coil tends to be higher than in plain coil. This phenomenon results in a relatively longer streamline and strong turbulence between the wall area and the core area, which increases heat transfer.
COP Improvement factors increase with decreasing pitch ribs. Ribs angle $20^{\circ}$ results in high COP Improvement Factors. Curvature coil $2.66 \mathrm{~m}^{-1}$ produces high COP Improvement Factors. The use of DDIR in low curvature coil / slinky-coil is recommended for practical applications of Ground Heat Exchanger, especially at low flowrate.

\section{Acknowledgements}

This research was supported by the "Renewable energy heat utilization technology and development project" of the New Energy and Industrial Technology Development Organization (NEDO), Japan.

\section{References}

[1] C. S. Chong, G. Gan, A. Verhoef, R. G. Garcia, and P. L. Vidale, "Simulation of Thermal Performance of Horizontal Slinky-loop Heat Exchangers for Ground Source Heat Pumps," Appl. Energy, vol. 104, pp. 603-610, 2013.

[2] B. Bouhacina, R. Saim, and H. F. Oztop, "Numerical Investigation of a Novel Tube Design for the Geothermal Borehole Heat Exchanger," Appl Therm Eng, vol. 79, pp. 153-162, 2015.

[3] J. Acuña and B. Palm, "Comprehensive Summary of Borehole Heat Exchanger Research at KTH," in Proceedings of Conference on Sustainable Refrigeration and Heat Pump Technology, 2009.

[4] Y. Wu, G. Gan, A. Verhoef, P. L. Vidale, and R. G. Gonzalez, "Experimental Measurement and Numerical Simulation of Horizontal-coupled Slinky Ground Source Heat Exchangers," Appl. Therm. Eng., vol. 30, pp. 2574-2583, 2010.

[5] S. Selamat, A. Miyara, and K. Kariya, "Numerical Study of Horizontal Ground Heat Exchangers For Design Optimization," Renew. Energy, vol. 95, pp. 561-573, 2016.

[6] M. H. Ali, K. Kariya, and A. Miyara, "Performance Analysis of Slinky Horizontal Ground Heat Exchangers for a Ground Source Heat Pump System," Resources, vol. 56, pp. 1-18, 2017.

[7] B. K. Hardik, P. K. Baburajan, and S. V. Prabhu, "Local Heat Transfer Coefficient in Helical Coils with Single Phase Flow," Int. J. Heat Mass Transf., vol. 89, pp. 522-538, 2015.

[8] G. Yoo, H. Choi, and W. Dong, "Fluid Flow and Heat Transfer Characteristics of Spiral Coiled Tube: Effects of Reynolds Number and Curvature Ratio," J. Cent. South Univ, vol. 19, pp. 471-476, 2012.

[9] J. Meng, "Enhanced Heat Transfer Technology of Longitudinal Vortices Based on Field-coordination Principle and its Application," Tsinghua University, 2003. (in Chinese)

[10] X. W. Li, J. A. Meng, and Z. Y. Guo, "Turbulent Flow and Heat Transfer in Discrete Double Inclined Ribs Tube," Int. J. Heat Mass Transf., vol. 52, pp. 962-970, 2009.

[11] N. Zheng, P. Liu, F. Shan, Z. Liu, and W. Liu, "Numerical Investigations of the Thermal-hydraulic Performance in a Ribgrooved Heat Exchanger Tube Based on Entropy Generation Analysis," Appl. Therm. Eng., vol. 99, pp. 1071-1085, 2016.

[12] T. H. Ariwibowo, G. Kuriyama, K. Kariya, and A. Miyara, "Numerical Analysis of Thermo-Hydraulic Performance of Discrete Double Inclined Ribs on Low Curvature Coil in Laminar Flow for Ground Source Heat Pump System Application," in Proceedings of 15th Asia Pacific Conference on the Built Environment 5R Technology for Building Environment, 2019.

[13] T. H. Ariwibowo, A. Miyara, and K. Kariya, "Thermal and Hydraulic Performance Simulation of Curved Tube with Discrete Ribs Heat Exchanger for Ground Heat Pump System," in Proceedings of 53rd Joint Air Conditioning and Refrigeration Conference, 2019.

[14] T. H. Ariwibowo, A. Miyara, and K. Kariya, "Consideration of Double Discrete Inclined Ribs in Low Curvature Coil for GSHP System," Int. J. Sustain. Green Energy, vol. 8, pp. 56-64, 2019.

[15] G. Kuriyama, T. H. Ariwibowo, K. Kariya, and A. Miyara, "Heat Transfer and Pressure Drop Characteristics of Curved Tube with Discrete Ribs for Ground Source Heat Exchanger," in Proceedings of the 2019 Refrigeration and Air Conditioning Society of Japan Annual Meeting, 2019. (in Japanese) 
[16] F. Menter, M. Kuntz, and R. Langtry, "Ten Years of Industrial Experience with the Sst Turbulence Model," Turbul. Heat Mass Transf, vol. 4, 2003.

[17] V. Holm'en, "Methods for Vortex Identification," Lund University, 2012.

[18] ANSYS® Academic Research, Release 17.2, Help System, Fluent User's Guide. ANSYS, Inc., 2016.

[19] H. Ito, "Friction Factor for Turbulent Flow in Curved Tube," $J$. Basic Eng., vol. 81, pp. 123-134, 1959.
[20] Jalaluddin and A. Miyara, "Thermal Performance and Pressure Drop of Spiral-tube Ground Heat Exchangers for Ground-source Heat Pump," Appl. Therm. Eng., vol. 56, pp. 630-637, 2015.

[21] V. Gnielinski, "On Heat Transfer in Tubes," Int. J. Heat Mass Transf., vol. 63, pp. 134-140, 2013.

[22] J. P. Abraham, E. M. Sparrow, and W. J. Minkowycz, "Internalflow Nusselt Numbers for The Low-reynolds-number End of the Laminar-to-turbulent Transition Regime," Int. J. Heat Mass Transf., vol. 54, pp. 584-588, 2011. 